\title{
A combined experimental with simulation approach to calibrated 3D strain measurement using shearography
}

\author{
Dênis T. Goto ${ }^{\mathrm{a}, \mathrm{b}}$, Roger M. Groves ${ }^{\mathrm{a}}$ \\ ${ }^{a}$ Delft University of Technology, Optical Non-Destructive Testing Laboratory, Faculty of Aerospace \\ Engineering, Kluyverweg 1, 2600 GB Delft, The Netherlands \\ ${ }^{\mathrm{b}}$ Universidade Federal de Santa Catarina, Laboratório de Metrologia e Automatização, Departamento \\ de Engenharia Mecânica, CEP 88040-970, Florianópolis, Brazil
}

\begin{abstract}
This paper is concerned with the development of a calibrated 3D shearography strain measurement instrument, calibrated iteratively, using a combined mechanical-optical model and specially designed test objects. The test objects are a cylinder loaded by internal pressure and a flat plate under axial load. Finite element models of the samples, combined with optical models of the shearography system, allow phase maps to be simulated for subsequent comparison with experimental phase maps from the shearography instrument. The algorithm to extract the strain maps from the phase maps includes an error compensation for in-plane strains on curved surfaces, measurement channel redundancy, variations in the shear magnitude due to object shape and the optical characteristics of the imaging system. The improvement introduced by the error compensation techniques is verified by the opto-mechanical simulation and its effect is demonstrated experimentally on maps of displacement gradient.
\end{abstract}

Keywords: 3D Shearography, strain measurement, error analysis.

\section{INTRODUCTION}

Advanced optical measurement techniques are in common use for measurement applications in high technology industries. Shearography ${ }^{1}$, a technique closely related to holography, uses a speckle shearing interferometer to give a direct sensitivity to the surface displacement gradient, from which strain information can be extracted. The direction of the gradient is defined by the shearing direction and in the general case shearography displays sensitivity to gradient of displacement in all three Cartesian directions. By careful selection of the relative positions of the interferometer head and the laser source, it is possible to minimize sensitivity to certain components. For example illumination and viewing directions nearly normal to the object surface maximize sensitivity to the out-of-plane displacement gradient. A multiple channel shearography system is required to isolate in-plane displacement gradients. The most common configuration is the symmetric dual beam setup ${ }^{1}$ which sequentially measures the object with each of the two illumination directions. The two phase maps obtained are subtracted to determine the in-plane displacement gradient. As an alternative, shearography instruments with three, or more, measurement channels (3D shearography systems) do not require accurate symmetry as they are able to isolate gradients of displacement along all three Cartesian directions. For this last case, strain measurement using both the multiple laser ${ }^{2,3,4}$ and the multiple camera configuration ${ }^{5}$ is possible.

A number of studies of errors in quantitative strain measurement using shearography have been published together with techniques for error compensation. One source of error is associated with the assumption that shearography measures displacement gradient, while in fact it is the difference of displacements of two neighbouring points that is being measured ${ }^{6}$. Errors are also introduced by the assumption of a constant shear distance, as variable shear is inherent to the Michelson interferometer ${ }^{7}$ which is the most used shearing device. Some studies propose methods for shear magnitude measurement, including digital image correlation ${ }^{8}$. By adopting a constant sensitivity vector, some geometry parameters are being neglected, such as the finite size of object and camera sensor ${ }^{9}$. The use of an expanded illumination beam ${ }^{10,11}$, which is suitable for large objects, introduces an even larger variation of the sensitivity vector. In the case of 3D shearography systems, a matrix transformation is required and the level of uncertainty of this matrix operation may be measured by the conditioning number ${ }^{12}$. This error depends on the relative positions of the measurement channels. An object with a curved surface introduces a variable shear magnitude due to the variable misalignment of the sensor plane with the object surface. A correction may be adopted for this if the object shape is known, or as an alternative shearography may be used to measure the object shape and slope ${ }^{13}$.

Speckle 2010: Optical Metrology, edited by Armando Albertazzi Goncalves Jr., Guillermo H. Kaufmann, Proc. of SPIE Vol. 7387, 73871 · @ 2010 SPIE · CCC code: 0277-786X/10/\$18 · doi: 10.1117/12.870572 
This paper addresses the measurement of strain on a curved surface using a 3D shearography system with four cameras and one laser source. Error sources are investigated by the development of an opto-mechanical model of a 3D shearography instrument, described in more detail previously ${ }^{14}$, and by comparison with experimental results. The following error sources are addressed in this paper. One is that the object surface cannot be assumed to be always parallel to the sensor plane, because the displacement gradients are calculated along the shear direction, which is determined by the camera sensor plane. If the object surface is parallel to the camera sensor plane, the displacement gradients may be assumed to be the in-plane gradients. Both the object curvature and the viewing geometry can contribute to this misalignment. The variation in shear magnitude due to object shape and optical distortion is addressed. Image processing software with compensations for these errors is described and experimental and simulated phase maps are compared.

\section{SHEAROGRAPHY INSTRUMENT AND CALIBRATION SAMPLE}

The shearography instrument modelled and used for the experimental measurements has four cameras arranged at the tips of a cross according to Figure 1-A. Interferometers 1 and 3 are $400 \mathrm{~mm}$ laterally from the centre of the cross while the interferometers 2 and 4 are $400 \mathrm{~mm}$ vertically from the centre of the cross. The laser source is placed $120 \mathrm{~mm}$ below the centre of the cross. These camera positions minimize the errors in the matrix transformation required to isolate the displacement gradients ${ }^{2}$. The distance between the object and the cross structure is $1 \mathrm{~m}$. Although three cameras are enough to characterize all components of in plane strain, one extra camera allows multiple measurement channel combinations and a better confidence level is then obtained ${ }^{12}$.

A thin wall aluminium cylinder is used as calibration sample. The cylinder is $400 \mathrm{~mm}$ in length, with an external diameter of $190 \mathrm{~mm}$ and a wall thickness of $5 \mathrm{~mm}$. It was manufactured by rolling an aluminium plate and welding the seam. To complete the construction aluminium end caps were welded to the ends of the cylinder. Oil is pumped into the cylinder by a mechanical pump and the loading magnitude is controlled by a pressure gauge.
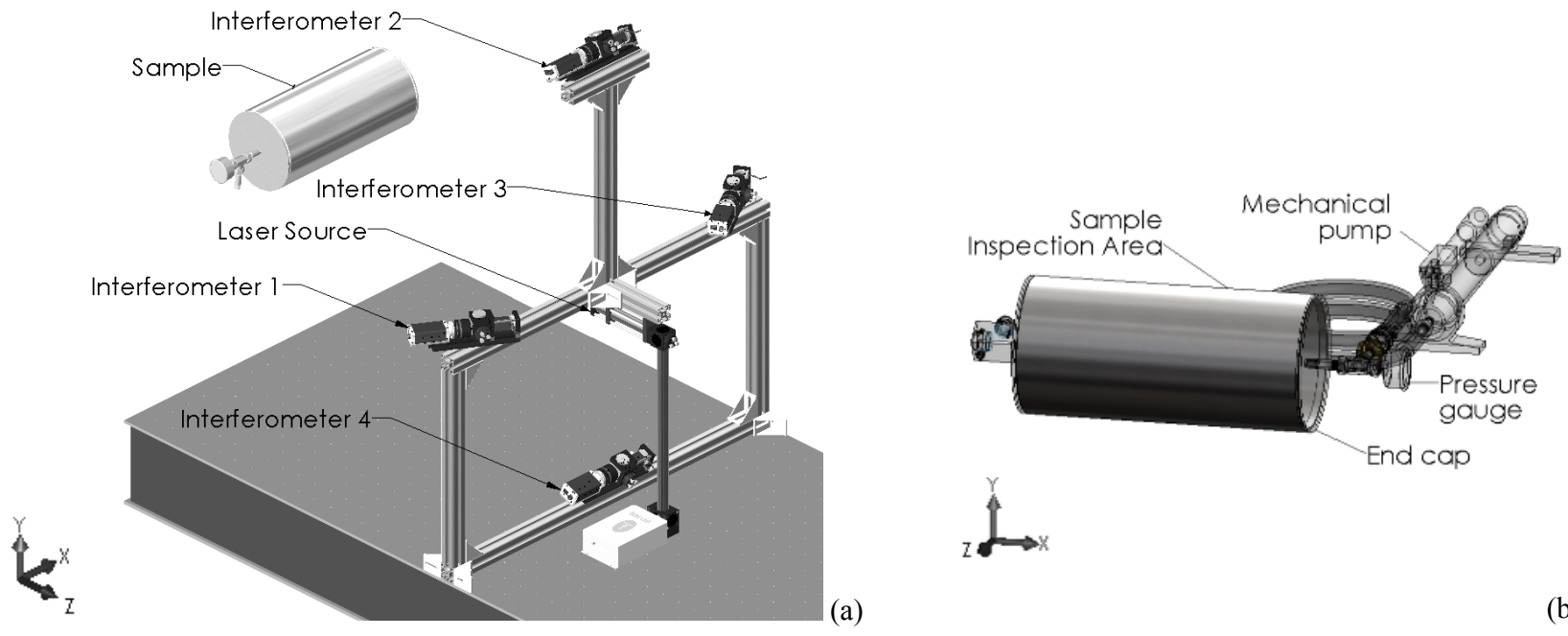

Figure 1. (a) 3D Shearography instrument, showing the locations of the laser and the interferometers. (b) Cylinder sample.

The sample was designed to provide a nearly constant strain field across a large area on the sample surface and in the measurement range of shearography. An analytical model provided strain values which are summarized in the Table 1. Uncertainties in the sample material properties, manufacturing tolerances and loading inaccuracy were included in the model and allowed an estimate of the error in the calculated strain. The calculated strain was measured by electrical strain gauges attached at four different points of the object and good agreement was achieved. Also, the strain gauges demonstrated that the strain field is nearly constant along the sample.

Table 1. Expected strain according to the analytical model.

\begin{tabular}{|lrl}
\multicolumn{3}{l}{ Cylinder sample in-plane strain } \\
Longitudinal & Analytical model & Strain gauges (mean) \\
Tangential & $(4.31 \pm 1.58) \cdot 10^{-5}$ & $4.50 \cdot 10^{-5}$ \\
& $(2.12 \pm 0.70) \cdot 10^{-4}$ & $2.82 \cdot 10^{-4}$ \\
\hline
\end{tabular}




\section{PHASE MAPS}

\subsection{Simulated phase maps}

From an optical point of view it follows that a phase map obtained with shearography is related to the optical pathlengh difference between the laser source (S) and the camera (C) during loading of the object.

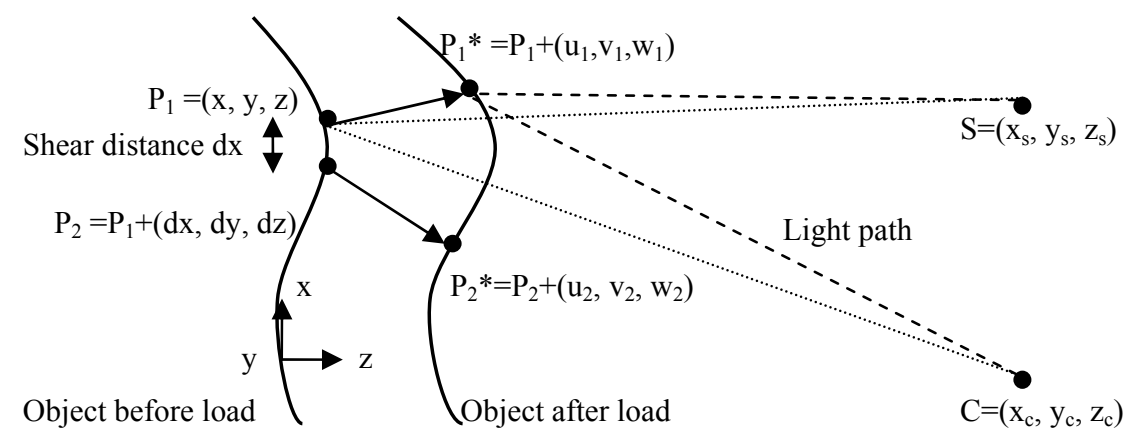

Figure 2. Schematic of the optical and engineering mechanics interpretation of the phase change. $\mathrm{S}$ is the source position, $\mathrm{P}_{1}$ and $\mathrm{P}_{2}$ are reference points on the object surface and $\mathrm{C}$ is the camera position. $\mathrm{P}_{1} *$ and $\mathrm{P}_{2} *$ are points on the object surface after applying a deformation.

A global coordinate system is defined with its origin on the object surface and with the $\mathrm{z}$ direction normal to the surface. $\mathrm{P}_{1}$ and $\mathrm{P}_{2}$ are reference points on the object surface and $\mathrm{C}$ is the camera position. $\mathrm{P}_{1}{ }^{*}$ and $\mathrm{P}_{2} *$ are points on the object surface after applying a deformation. Position coordinates are denoted by $(\mathrm{x}, \mathrm{y}, \mathrm{z})$ and displacements are denoted by $(\mathrm{u}, \mathrm{v}, \mathrm{w})$, with the subscript indicating the point referred to. The shear is expressed by (dx,dy,dz).

$$
\Delta \phi=\frac{2 \pi}{\lambda}\left[\left(\overline{S P_{1}^{*} C}-\overline{S P_{1} C}\right)-\left(\overline{S P_{2}^{*} C}-\overline{S P_{2} C}\right)\right]
$$

$\Delta \phi$ is the phase change and $\lambda$ is the optical wavelength. Optical pathlengths are indicated by $\overline{S P_{1}^{*} C}, \overline{S P_{1} C}, \overline{S P_{2}^{*} C}$ and $\overline{S P_{2} C}$. Equation (1) is the main equation of the optical model used to simulate the shearography system. The source position is the same for all measurement channels, but each channel has its own camera position. Object surface position before and after loading are required and these are obtained from the finite element model of the samples. Finite element analyses were performed using Abaqus (version 6.8) on a Pentium D 3,40GHz with 2GB of RAM. A 2D model consisting of one quarter of the cylinder was prepared with adequate symmetry boundary conditions. Pressure loading was directly applied to the internal surface of the Finite element model.

\subsection{Experimental phase maps}

To perform a measurement with shearography, reference interferograms are recorded and a phase shift algorithm ${ }^{15}$ is applied to determine the difference of phase at each pixel. This forms the reference map of difference of phase. The object is then loaded to a second state, further interferograms are recorded and the phase shift algorithm is again used to determine the map of difference of phase at this second loading state. This is the signal map of difference of phase. Reference and signal maps are then correlated by subtraction to yield a phase map. In this paper, temporal phase shifting with three phase steps is used to calculate the map of phase difference $(\phi)$ using equation $(2)$ :

$$
\phi=\arctan \left[\sqrt{3} \frac{I_{3}-I_{1}}{2 I_{2}-I_{1}-I_{3}}\right]
$$

Where $I_{1}, I_{2}$ and $I_{3}$ are the recorded interferograms with a $2 \pi / 3$ phase step between each pair. An extended arctan function is used to obtain phase values in the range from $-\pi$ to $+\pi$. To minimise phase unwrapping errors, the sample is loaded in small steps and a sequence of phase maps, $\Delta \phi_{i}$, are calculated by subtracting consecutive maps of phase difference.

$$
\Delta \phi_{i}=\phi_{i}-\phi_{i-1}
$$


This phase map is in the range $[-2 \pi, 2 \pi]$. A correction ${ }^{16}$ defined by equation (4) is applied to correct the range to $[-\pi, \pi]$.

$$
\begin{array}{ccc}
\Delta \phi & \text { if } & -\pi \leq \Delta \phi \leq \pi \\
\Delta \phi=\Delta \phi-2 \pi & \text { if } & \Delta \phi>\pi \\
\Delta \phi+2 \pi & \text { if } & \Delta \phi<-\pi
\end{array}
$$

The corrected phase map is smoothed by a sine-cosine filter ${ }^{1}$. If the phase does not experience a variation outside the range $[-\pi, \pi]$ in each individual phase map $\Delta \phi_{i}$, than the phase map that represents the displacement gradient of the full loading range may be obtained by adding all partial phase maps. In this case, phase unwrapping is avoided ${ }^{17}$.

In order to process the final phase maps to obtain strain maps, images from multiple measurement channels must be correlated. In the case of a multiple camera shearography system, each camera sees the object from a different viewing angle, which introduces perspective distortion. Also, when the object is not flat, co-registration becomes difficult due to the perspective distortion. Ideally, the images from each camera should be mapped on a cylindrical surface for a perfect co-registration.

In this paper, perspective distortion correction is performed using a tool for image calibration available in Labview. A calibration pattern composed of a grid of black round dots with a known distance between consecutive dots is placed in front of the object and imaged with each camera of the shearography sensor without any applied shear. The algorithm recognizes the dot pattern and builds a correction function. The correction function obtained for each camera is then applied to the final phase maps to eliminate the perspective distortion.

A simple cylinder mapping algorithm that translates rows of pixels was used to reduce the effect of the flat shape acquired by the object. The correction was performed for cameras 1 and 3 (see Figure 1-a) as the distortion is larger for this cameras.
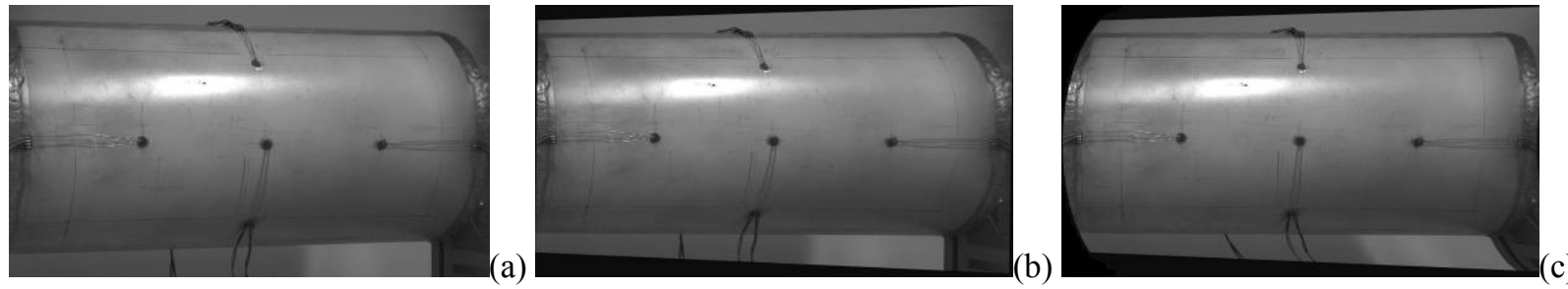

Figure 3. (a) is the original image from camera 1 with perspective distortion, (b) the image after the correction for perspective distortion and (c) the image after translation of rows of pixels.

\section{FROM PHASE MAPS TO STRAIN MAPS}

\subsection{D strain calculation}

By considering Figure 2, the engineering mechanics interpretation of the phase map is:

$$
\Delta \phi=\frac{2 \pi}{\lambda} \vec{k} \cdot\left[\begin{array}{c}
u_{2}-u_{1} \\
v_{2}-v_{1} \\
w_{2}-w_{1}
\end{array}\right] \cong \frac{2 \pi}{\lambda} \vec{k} \cdot\left[\begin{array}{l}
\partial u / \partial x \\
\partial v / \partial x \\
\partial w / \partial x
\end{array}\right] \cdot d x
$$

Where $d x$ is the shear distance and $\partial u / \partial x, \partial v / \partial x$ and $\partial w / \partial x$ are the displacement gradients. Equation (5) may also be formed with the shear in y direction and in this case the displacement gradients are along y direction. The vector $\vec{k}$ is known as sensitivity vector and is approximated by:

$$
\vec{k}=\frac{\overrightarrow{P_{1} S}}{\left|\overrightarrow{P_{1} S}\right|}+\frac{\overrightarrow{P_{1} C}}{\left|\overrightarrow{P_{1} C}\right|}
$$

A 3D shearography system with three cameras is capable of generating three maps of phase difference for each shearing direction, which allows isolation of each displacement gradient by solving the coordinate transformation ${ }^{18}$ : 


$$
\left[\begin{array}{l}
\partial u / \partial x \\
\partial v / \partial x \\
\partial w / \partial x
\end{array}\right]=\frac{\lambda}{2 \pi}\left[\begin{array}{l}
\vec{k}_{\text {cam } 1} \\
\vec{k}_{\text {cam } 2} \\
\vec{k}_{\text {cam } 3}
\end{array}\right]^{-1}\left[\begin{array}{l}
\Delta \phi_{x, \text { cam } 1} \\
\Delta \phi_{x, \text { cam } 2} \\
\Delta \phi_{x, \text { cam } 3}
\end{array}\right] \frac{1}{d x}=\frac{\lambda}{2 \pi} M^{-1}\left[\begin{array}{l}
\Delta \phi_{x, \text { cam } 1} \\
\Delta \phi_{x, \text { cam } 2} \\
\Delta \phi_{x, \text { cam } 3}
\end{array}\right] \frac{1}{d x}
$$

$\mathrm{M}$ is $3 \times 3$ matrix defined by concatenating the components of the sensitivity vector for each camera. Subscripts indicate the camera and shear direction. By applying a shear in the y direction, the gradients $\partial u / \partial y, \partial v / \partial y, \partial w / \partial y$ may be obtained. In-plane strain maps are then calculated by:

$$
\begin{gathered}
\varepsilon_{x}=\partial u / \partial x \\
\varepsilon_{y}=\partial v / \partial y \\
\varepsilon_{x y}=\frac{1}{2}\left(\frac{\partial u}{\partial y}+\frac{\partial v}{\partial x}\right)
\end{gathered}
$$

Where $\varepsilon_{\mathrm{x}}$ and $\varepsilon_{\mathrm{y}}$ are axial strains in the $\mathrm{x}$ and $\mathrm{y}$ directions respectively and $\varepsilon_{\mathrm{xy}}$ is the shear strain.

\subsection{Sensitivity vector correction for curved object}

Equation (5) describes the approximation of the displacement gradient by the difference of displacement between two points on the object separated by the shear distance. The gradients are along the Cartesian directions of a pre-defined global coordinate system (see Figure 2). The global coordinate system is defined to have the xy plane aligned with the camera sensor plane and with the object surface. In this way, the shear distance along the camera sensor plane (in pixels), applied by means of the Michelson interferometer, may be directly associated with the distance (in $\mathrm{mm}$ ) that separates the two points on the object surface. As the object surface is supposed to be aligned with the xy plane, then the approximation described by equation (5) may be used to describe in-plane displacement gradients and thus in-plane strain. However, in case the object surface is not aligned with the xy plane, then the approximation of equation (5) may not be used, as the displacement gradient will be associated with the difference of displacements of points with a $\mathrm{z}$ offset.

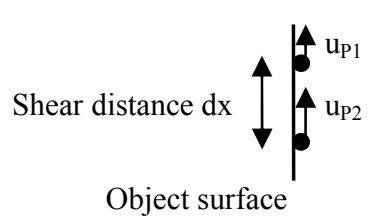

Object surface

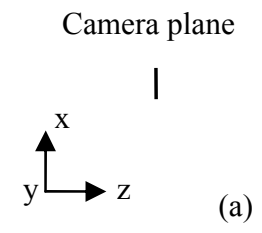

(a)

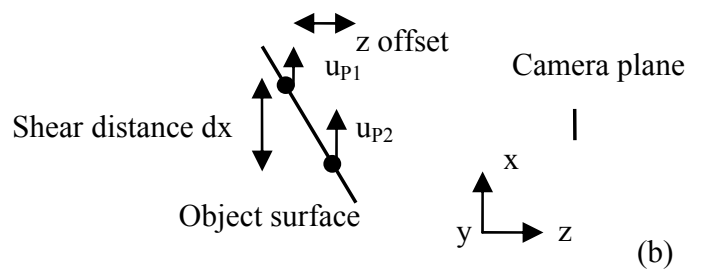

$\frac{d u}{d x} \neq \frac{u_{P 1}-u_{P 2}}{d x}$

Figure 4 - (a) The shearography approximation is suitable when the object surface is parallel to camera sensor plane (C). (b) A correction is required when the object surface is not parallel to the camera sensor plane.

According to equation (1), for fixed relative positions of the camera, the object and the laser source, as well as for a fixed laser wavelength, the phase map is only dependent on the object deformation. This same object deformation may be described in any coordinate system, in other words, equation (5) describes this phase map independently of the adopted coordinate system. Thus, equation (7) may be used to obtain the displacement difference along any direction. The directions are chosen by selecting the adequate orientation of the coordinate system. By selecting at each point on the object surface, a local coordinate system in which the $\mathrm{z}$ coordinate is normal to the object surface; it is possible to calculate in-plane difference of displacements, which can then be associated with in-plane displacement gradient and inplane strain. From the practical point of view, the positions of all cameras and the laser are determined using the global coordinate system, sensitivity vectors are calculated in this coordinate system and then rotated to the local coordinate system. The coordinate rotation for the cylinder sample is defined by: 


$$
\vec{k}_{\text {local }}=\left(\begin{array}{ccc}
1 & 0 & 0 \\
0 & \cos \left(e_{l}\right) & -\sin \left(e_{l}\right) \\
0 & \sin \left(e_{l}\right) & \cos \left(e_{l}\right)
\end{array}\right) \cdot \vec{k}_{\text {global }}
$$

Where $\mathrm{e}_{1}$ is the elevation angle measured from the positive $\mathrm{z}$ direction to the positive $\mathrm{y}$ direction of the global coordinate system.

\subsection{Shear magnitude measurement and correction for curved object}

The shear distance is the distance in $\mathrm{mm}$ along the object surface between the points that are being correlated on the camera sensor plane. The shear is applied on the camera sensor plane and is measured in pixels. Usually, a known distance along the object is used and a ratio pixel $/ \mathrm{mm}$ is defined in order to calculate the shear distance in $\mathrm{mm}$ along a virtual plane placed at the same position of the object. However, the use of non ideal optical components introduces some variation of the shear distance measured in pixels. In order to eliminate this distortion, a random greyscale pattern was placed in front of the object and imaged with the shearography sensor twice, with each image recorded with one of the mirrors inside the Michelson interferometer blocked. As a result, two images were obtained where the greyscale pattern appears to be at different positions. 2D digital image correlation software, developed in-house by R.-J. Lemmen ${ }^{19}$, was used to generate a map of shear distances (in pixels). A known distance along the recorded images was used to calibrate a global ratio pixel $/ \mathrm{mm}$ and to generate the correspondent map of shear distances (in $\mathrm{mm}$ ). This process was performed for all measurement channels.

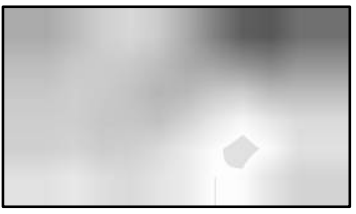

(a)

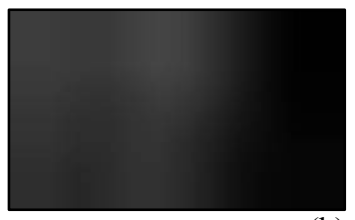

(b)

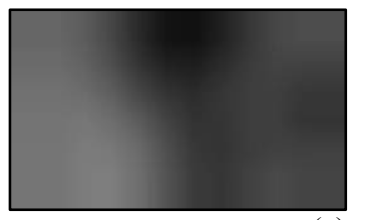

(c)

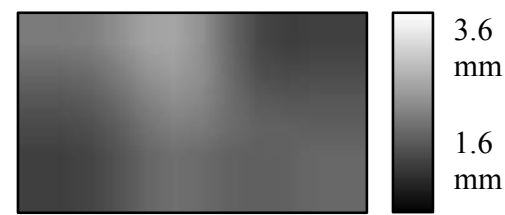

(d)

Figure 5 - Maps of shear distance along the horizontal direction. (a) Camera 1, (b) camera 2, (c) camera 3 and (d) camera 4.

In the case of the measurement of a flat plate, the generated map of shear distance (in mm) corresponds to the in-plane shear distances. However in the case of curved objects, additional corrections are required. For the cylinder sample, there is shear variation only for the shear along the vertical direction on the recorded images, as this direction coincides with the tangential direction of the cylinder. Due to the simple form of the cylinder, a correction based on trigonometry was possible.

$$
d y_{\text {surf }}=d y \cdot \cos \left(e_{l}\right)
$$

Where $d y_{\text {surf }}$ is the in-plane shear distance, dy is the shear distance measured by digital image correlation and $e l$ is the elevation angle.The shear in the horizontal direction does not require correction as this direction is aligned with the cylinder axis. The variation in the pixel $/ \mathrm{mm}$ ratio due to the variation of the distance between the camera and the cylinder surface was not considered.

After performing all corrections, equation (7) becomes

$$
\left[\begin{array}{l}
\partial u^{\prime} / \partial x^{\prime} \\
\partial v^{\prime} / \partial x^{\prime} \\
\partial w^{\prime} / \partial x^{\prime}
\end{array}\right]=\frac{\lambda}{2 \pi}\left(M^{\prime}\right)^{-1}\left[\begin{array}{c}
\Delta \phi_{x, c a m 1} / d x_{c a m 1} \\
\Delta \phi_{x, \text { cam } 2} / d x_{c a m 2} \\
\Delta \phi_{x, \text { cam } 3} / d x_{c a m 3}
\end{array}\right]
$$

Where $\partial u^{\prime} / \partial x^{\prime}, \partial v^{\prime} / \partial x^{\prime}$ and $\partial w^{\prime} / \partial x^{\prime}$ are the in-plane displacement gradients, $M^{\prime}$ is the sensitivity matrix obtained after rotation of the sensitivity vectors to the local coordinate system and $d x_{\text {caml }}, d x_{\text {cam } 2}$ and $d x_{\text {cam } 3}$ are the maps of in-plane shear distance. Equations (8), (9) and (10) are used to calculate the in-plane strain. 


\section{RESULTS AND DISCUSSION}

\subsection{Phase maps}

The opto-mechanical simulation was used to calculate the expected phase maps for each measurement channel. This approach was used so that a comparison can be made without considering errors in the 3D strain calculation. The simulated and experimental phase maps obtained have similar characteristics, but the experimental fringes show some distortions. This is expected to be due to manufacturing tolerances. The edges of the sample were welded to the endcaps. Materials properties and dimensional tolerances are greatly affected by the welding process, thus the simulated phase maps are not accurate enough to allow quantitative comparison for these areas of the sample. For this reason, regions of interest (ROI) marked by the black rectangles in Figure 6 and Figure 7 are areas suitable for quantitative analysis. The regions indicated by the arrows are areas where the recorded intensity was too bright and phase extraction was not successful. The "x" marks the fringe that has the zero phase.

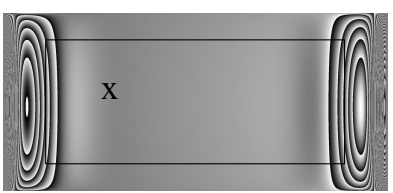

(a)

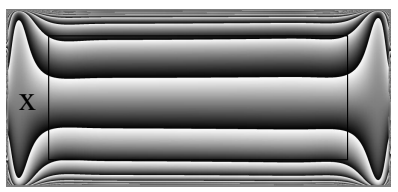

(e)

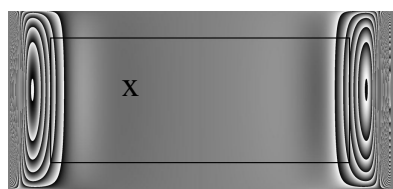

(b)

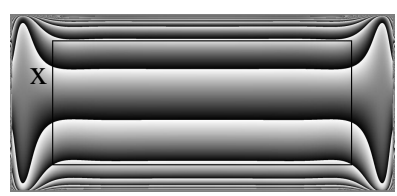

(f)

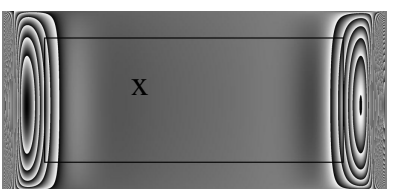

(c)

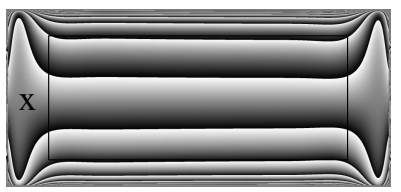

(g)

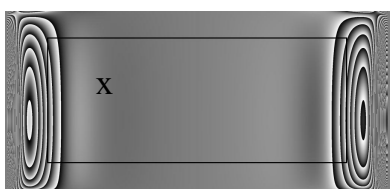

(d)

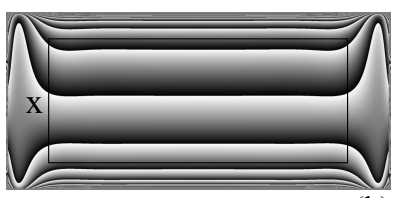

(h)

Figure 6. Simulated phase maps of the cylinder sample with $2.3 \mathrm{~mm}$ x shear: (a) camera 1, (b) camera 2, (c) camera 3 and (d) camera 4. 1.8mm y shear: (e) camera 1, (f) camera 2, (g) camera 3 and (h) camera 4.

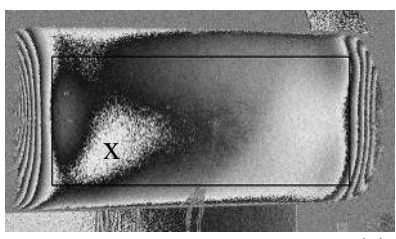

(a)

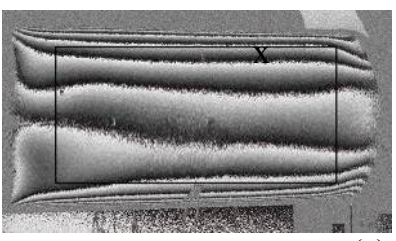

(e)

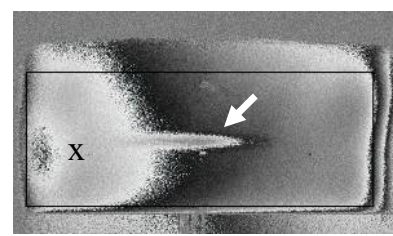

(b)

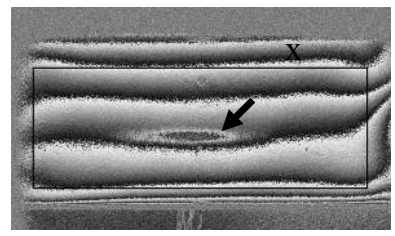

(f)

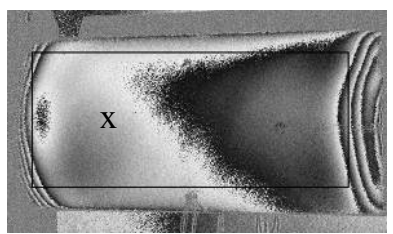

(c)

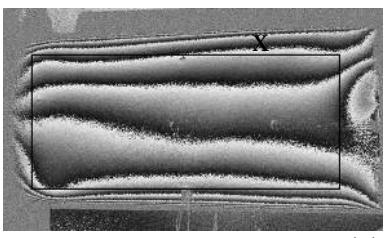

(g)

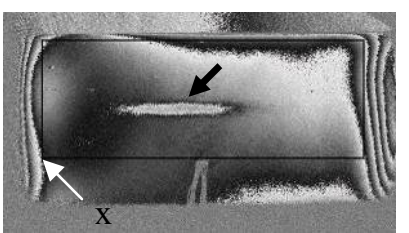

(d)

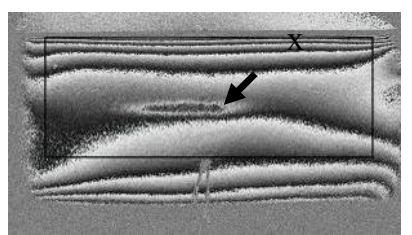

(h)

Figure 7. Measured phase maps of the cylinder sample with $2.3 \mathrm{~mm}$ of nominal x shear: (a) camera 1, (b) camera 2, (c) camera 3 and (d) camera 4. $1.8 \mathrm{~mm}$ nominal y shear: (e) camera 1, (f) camera 2, (g) camera 3 and (h) camera 4.

The unwrapped phase maps are more suitable than the wrapped phase maps for a numerical comparison due to the absence of fringes. The section of the image within the defined ROI of the experimental phase maps were co-registered and subtracted from the corresponding area of the simulated phase maps. Considering the phase maps with shear in the horizontal direction, the simulated phase maps are much more uniform than the experimental maps, the difference is within the range $[-2 \pi, 0]$ for most of the phase map. Considering the maps with shear in the vertical direction, the difference is within $[2 \pi, 4 \pi]$, in other words, the position of the zero order fringe in the experimental phase maps does not match with the positions of the zero order fringe in the simulated phase maps. This difference might be associated with a low correlation between the fixtures used for the real sample and the boundary conditions used to describe the fixtures in the finite element simulation. This issue is under further investigation. 


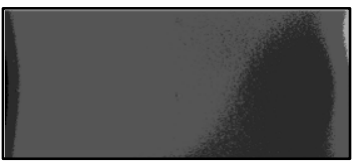

(a)

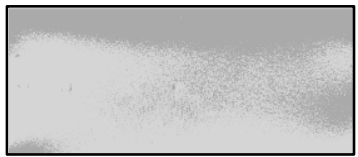

(e)

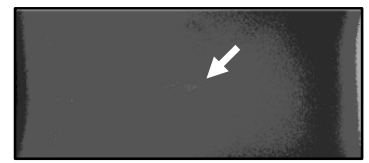

(b)

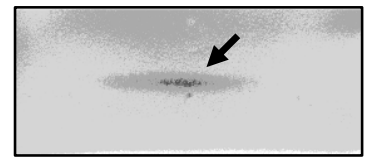

(f)

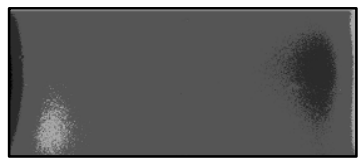

(c)

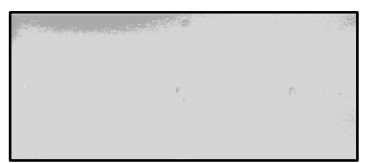

(g)

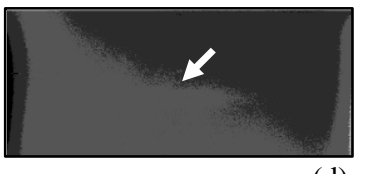

(d)

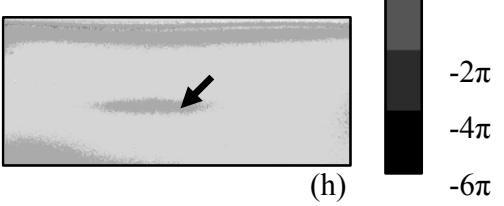

Figure 8 - Difference between simulation and experimental phase maps (only for the region of interest defined in Figure 7): for $\mathrm{x}$ shear (a) camera 1, (b) camera 2, (c) camera 3 and (d) camera 4. For y shear: (e) camera 1, (f) camera 2, (g) camera 3 and $(\mathrm{h})$ camera 4 . The arrows indicate areas where the phase extraction failed due to saturation of the recorded interferograms.

\subsection{Strain maps from simulated phase maps}

The shearography algorithm to calculate strain maps from the phase maps was applied to the simulated phase maps. The coordinate rotation of the sensitivity vectors introduces a large difference for the bottom and top edges of the image, which are areas where the misalignment of the sample surface with the camera is larger. The improvement obtained with the shape correction is visible in the maps of normalized difference $(\Delta)$ as defined by the equation below, where $\varepsilon_{\text {shearography }}$ is the strain calculated by the shearography algorithm and $\varepsilon_{F E M}$ is the strain from the finite element simulation.

$$
\Delta=\frac{\varepsilon_{\text {shearography }}-\varepsilon_{F E M}}{\varepsilon_{F E M}}
$$

Without the sensitivity vector correction, the strain maps display an error of $5 \%$ for a misalignment of $27^{\circ}$ between the global xy plane and the object surface. The error increases with a higher misalignment.

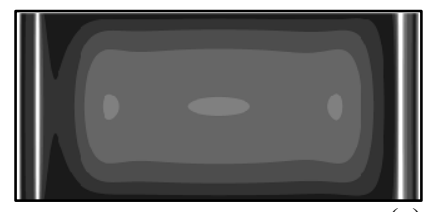

(a)

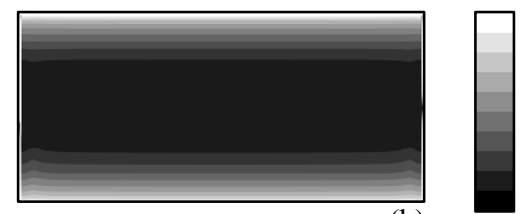

(b) $\geq 100 \%$

$50 \%$

$5 \%$

Figure 9. Comparison of the strain maps obtained from simulated phase maps and the finite element simulated strain.

Without the sensitivity vector correction. (a) Longitudinal and (b) tangential strain.

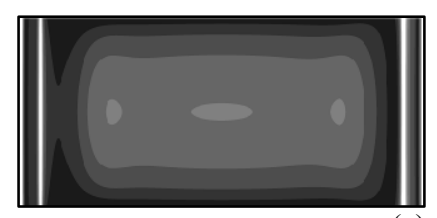

(a)

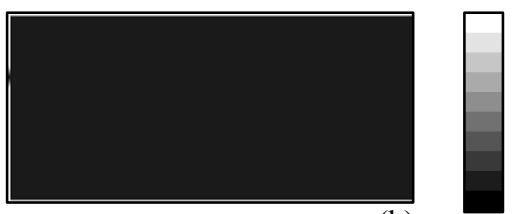

(b)

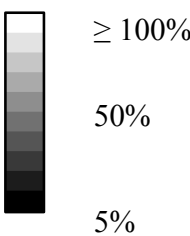

$5 \%$

Figure 10. Comparison of the strain maps obtained from simulated phase maps and the finite element simulated strain. With the sensitivity vector correction. (a) Longitudinal and (b) tangential strain.

\subsection{Experimental maps of displacement gradient}

Considering Figure 11, as the cylinder is loaded by internal pressure, the surface displacement is mostly along the radial direction. Its component along the global $\mathrm{z}$ direction assumes a circular distribution. Thus, the displacement gradient map is expected to display some variation. 


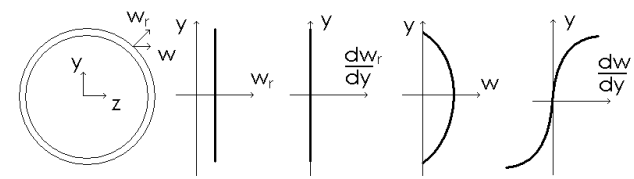

Figure 11. Schematic of the radial displacement and its component along the global $\mathrm{z}$ direction.

By applying the coordinate rotation to the sensitivity vectors, the displacement gradient calculated using equation (13) may be considered as the gradient of the radial displacement. Thus the displacement gradient map is expected to display low variation. Figure 12-a demonstrates the map of out-of-plane displacement gradient, adopting constant shear magnitude and without the rotation of the sensitivity vectors, while Figure 12-b was obtained with rotation of the sensitivity vectors. The rotation of the sensitivity vectors generates nearly zero displacement gradient even for the bottom and top areas of the image, where the misalignment of the sample surface with the global xy plane is larger.
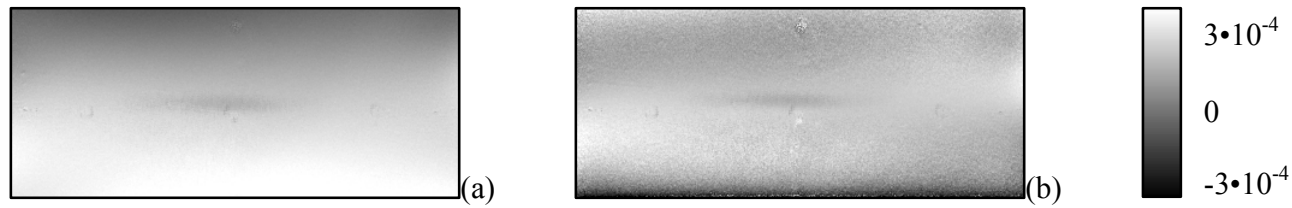

Figure 12. Map of out-of-plane displacement gradient ( $d w / d y)$ : (a) without rotation and (b) with rotation of the sensitivity vectors.

\section{CONCLUSIONS}

A combined optical mechanical simulation was used to generate phase maps which were then compared with experimental phase maps. Both simulation and experimental phase maps presented a similar shape, although there was a difference on the position of the zeroth fringe on the phase maps with vertical shear direction. This difference might be associated with a low correlation between the fixtures used to hold the sample and the boundary conditions used to describe the fixtures in the finite element simulation.

An algorithm to transform phase maps into strain maps that consider the influence of the sample curvature on the sensitivity vectors was described. The correction consists of a coordinate rotation. The sensitivity vectors are measured in a global coordinate system (with the $\mathrm{z}$ direction pointing to the shearography instrument) and then rotated to a local coordinate system where the $\mathrm{z}$ direction is always normal to the object surface. The correction allows measurement of inplane strain and its effect is noticeable for a large misalignment of the sample surface with the xy plane of the global coordinate system. The proposed correction was tested with simulated phase maps and we concluded that $5 \%$ of error is introduced in the strain field yielded by the shearography algorithm for a misalignment larger than $27^{\circ}$. The algorithm was also tested with experimental phase maps. The effectiveness of the algorithm was demonstrated on maps of displacement gradient.

Concerning experimental strain maps, a number of corrections already described in the literature were used (for the shear magnitude variation along the image, for the in-plane shear distance and for image dewarping and co-registration due to the camera positions), however the agreement between the measured strain map and the finite element simulated strain maps still needs to be improved. Accurate co-registration of images from different viewing angles has proven to be difficult when measuring non-planar objects especially due to the flat shape that the object acquires on the recorded interferograms. The improvement obtained with the sensitivity vector rotation was obscured by the cumulative effect of all other errors sources. Treatment of all found errors sources in this shearography system, including a better coregistration algorithm is the subject of current research.

\section{ACKNOWLEDGEMENTS}

The authors gratefully acknowledge TU Delft for the funding of this project and would like to thank Rik-Jan Lemmen for supplying software for the image correlation. D. T. Goto would like to thank the research team of the Laboratory for Metrology and Automation (UFSC) for the support during this research. 


\section{REFERENCES}

[1] Steinchen, W. Yang, L., [Digital Shearography], SPIE Press, Bellingham, Washington, 98227-0010 (2003).

[2] James, S. W. and Tatam, R. P., "Time-division multiplexed 3D shearography," Proc. SPIE 3744, 394-403 (1999).

[3] Waldner, S. Brem, S., "Compact shearography system for the measurement of 3D deformation," Proc SPIE 3745, 141-147 (1999).

[4] Kästle R, Hack E. Sennhauser U., "Multiwavelength shearography for quantitative measurements of twodimensional strain distributions," Appl. Opt. 38:1, 96-100 (1999).

[5] Francis, D., James, S. W. Tatam, R. P., "Surface strain measurement using multi-component shearography with coherent fibre-optic imaging bundles," Meas. Sci. Technol. 18, 3583-91 (2007).

[6] Labbe, F., Cordero, R. R., Martínez A., Rodrígues-Vera, R., "Measuring displacement derivatives by electronic speckle pattern shearing interferometry (ESPSI)" Meas. Sci. Technol. 16, 1677-83 (2005).

[7] Lee, J., Yoon, D., Kim, J., Vautrin, A., "Investigation of shear distance in Michelson interferometer-based shearography for mechanical characterization,” Meas. Sci. Techol. 19, 115303 (2008).

[8] Ng T. W., "Shear measurement in digital speckle shearing interferometry using digital correlation," Opt. Comm. 115, 241-244 (1995).

[9] Groves, R. M., Stephen, W., J., Rauph, P. T., "Strain measurement in curved industrial components using multicomponent shearography," Proc. SPIE 4398, 216-224 (2001).

[10] Farrant D. I., Petzing, J. N., "Sensitivity errors in interferometric deformation metrology," Appl. Opt 42:28, 5634-41 (2003).

[11] Wan Abdullah, W. S., Petzing, J. N., "Development of speckle shearing interferometer error analysis as an aperture function of wave front divergence," J. Mod. Opt.52:11, 1495-10 (2005).

[12] Groves, R. M., James, S. W., Tatam, R. P., "Multi-component shearography employing four measurement channels," Proc. SPIE 4933, 135-140 (2003).

[13] Groves, R. M., James, S. W., Tatam, R. P., "Shape measurement by source displacement in three-dimensional shearography”, Opt. Laser. Eng., 41, 621-634 (2004).

[14] Goto, D. T., Groves, R. M., 'Error analysis of 3D shearography using finite-element modelling', Proc. SPIE 7718, (in press), 2010.

[15] Dorrio B. V., Fernandez J. L., "Phase-evaluation methods in whole-field optical measurement techniques," Meas. Sci. Technol. 10, R33-R35 (1999).

[16] Huntley, J. M., “Automated fringe pattern analysis in experimental mechanics: a review," J. Strain Anal. 33, 105125 (1998).

[17] Groves, R.M., Furfari, D., Barnes, S.E., James, S.W., Fu, S., Irving, P.E., Tatam, R.P, "Full-field laser shearography instrumentation for the detection and characterization of fatigue cracks in titanium 10-2-3," J. ASTM Int. 3, JAI12757 (1996).

[18] James, S. W., Groves, R. M., Tatam, R. P., "Surface strain characterization using time-division-multiplexed 3D shearography," Proc. SPIE 4101, 389-398 (2000).

[19] Lemmen, H. J. K., Alderliesten, R. C., Benedictus, R., Hofstede, J. C. J., Rodi, R., "The power of digital image correlation for detailed elastic-plastic strain measurements," EMESEG '08 Conference Proceedings, 73-89 (2008). 